\title{
Leishmaniases in the European Union and Neighboring Countries
}

\author{
Eduardo Berriatua, Carla Maia, Cláudia Conceição, Yusuf Özbel, Seray Töz, Gad Baneth, \\ Pedro Pérez-Cutillas, Maria Ortuño, Clara Muñoz, Zarima Jumakanova, Andre Pereira, Rafael Rocha, \\ Begoña Monge-Maillo, Elkhan Gasimov, Yves Van der Stede, Gregorio Torres, Céline M. Gossner
}

\begin{abstract}
A questionnaire survey of animal and human health authorities in Europe revealed that leishmaniases are not notifiable in all countries with autochthonous cases. Few countries implement surveillance and control targeting both animal and human infections. Leishmaniases are considered emergent diseases in most countries, and lack of resources is a challenge for control.
\end{abstract}

$\mathrm{L}$ eishmaniases are endemic in humans and animals in part of the European Union (EU) and its neighboring countries. Leishmania species in this region are L. major, L. tropica, and the L. donovani complex species (including $L$. infantum and $L$. donovani sensu stricto). All cause cutaneous leishmaniasis (CL); visceral leishmaniasis (VL) is caused mainly by L. donovani complex species. There is evidence that the risk for leishmaniases is increasing in some EU and neighboring countries (1). We conducted a questionnaire survey to gather information on the epidemiologic situation, surveillance, prevention and control measures, and drivers of emergence of animal and human leishmaniases in this region during 2010-2020.

\section{The Study}

The survey included an animal leishmaniasis (AniL) questionnaire referring to L. infantum infections in domestic or wildlife hosts and a human leishmaniases

Author affiliations: Universidad de Murcia, Murcia, Spain (E. Berriatua, P. Pérez-Cutillas, M. Ortuño, C. Muñoz, Z. Jumakanova); Universidade NOVA de Lisboa, Lisbon, Portugal (C. Maia, C. Conceição, A. Pereira, R. Rocha); Ege University, Izmir, Turkey (Y. Özbel, S. Töz); The Hebrew University of Jerusalem, Rehovot, Israel (G. Baneth); Instituto Ramón y Cajal de Investigación Sanitaria, Madrid, Spain (B. Monge-Maillo); World Health Organization Regional Office for Europe, Copenhagen, Denmark. (E. Gasimov); European Food Safety Authority, Parma, Italy (Y. Van der Stede); World Organisation for Animal Health, Paris, France (G. Torres); European Centre for Disease Prevention and Control, Stockholm, Sweden (C.M. Gossner)

DOI: https://doi.org/10.3201/eid2706.210239
(HumL) questionnaire referring to infections by L. infantum, L. major, L. tropica and L. donovani s.s. (Appendix, https://wwwnc.cdc.gov/EID/article/27/6/210239-App1.pdf). The target audience was the national focal points (national institutes or ministries) of the European Centre for Disease Prevention and Control, the World Health Organization, the European Food Safety Authority, and the World Organisation for Animal Health in countries in which leishmaniases are endemic or those with confirmed or suspected presence of sand fly vectors (2). These countries were Albania, Algeria, Armenia, Austria, Azerbaijan, Belgium, Bosnia and Herzegovina, Bulgaria, Croatia, Cyprus, Czechia, Egypt, France, Georgia, Germany, Greece, Hungary, Israel, Italy, Jordan, Kosovo, Lebanon, Libya, Liechtenstein, Luxembourg, Malta, Moldova, Montenegro, Morocco, North Macedonia, Palestine, Portugal, Romania, Serbia, Slovakia, Slovenia, Spain, Tunisia, Turkey, and Ukraine (Figure 1). The questionnaires were administered electronically using the EU survey tool and shared on September 11, 2020 (3). Twenty-seven countries $(70 \%)$ replied to the AniL questionnaire and 24 countries $(60 \%)$ to the HumL questionnaires; 19 countries $(48 \%)$ replied to both (Table 1$)$.

We reviewed the countries' epidemiologic status with regards to autochthonous Leishmania spp. infections in animals and humans and clinical forms in humans. The mapping of the countries with autochthonous transmission matches previous published information with few discrepancies. For instance, according to the questionnaire, Bosnia and Herzegovina and Hungary do not have autochthonous canine leishmaniasis cases, although such cases have been described $(4,5)$. Human cases of leishmaniasis due to L. tropica were reported in Cyprus and Serbia and due to L. major in Georgia; however, none of the literature presents concurring evidence (Table 2).

Animal leishmaniases are notifiable in 17 countries and human leishmaniases in 20 countries (Table 1; Figure 2). In Palestine and Turkey, AniL is not notifiable despite a high prevalence among dogs $(6,7)$. Similarly, 


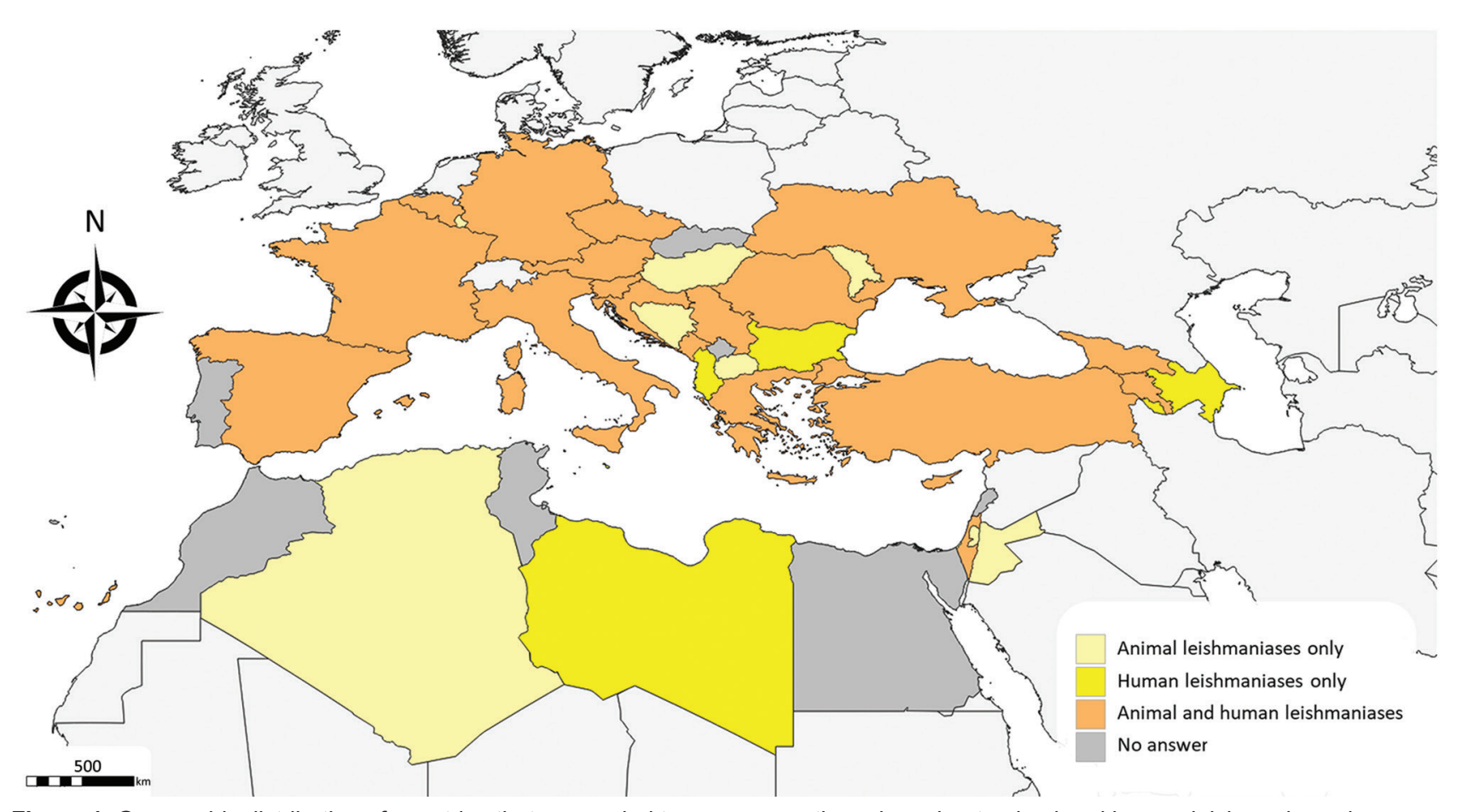

Figure 1. Geographic distribution of countries that responded to survey questionnaires about animal and human leishmaniases in Europe, 2020.

in France, neither AniL nor HumL are notifiable although the diseases are endemic in the south (8). Leishmaniases surveillance is not mandatory at the EU level which constitutes a limitation for successful control.

Seven countries conduct AniL surveillance (Table 1), indicative of its low priority among the animal health authorities. The target animal population for surveillance included symptomatic and asymptomatic dogs in Armenia, Cyprus, Italy, Spain, and Ukraine; we also studied wildlife in leishmaniasis foci in Spain. Testing subclinically infected dogs indicated awareness of their role as reservoirs of the parasite (1). Similarly, wild lagomorphs were the main reservoir of L. infantum in a HumL outbreak in Madrid in Spain (9). Surveillance of HumL is conducted in 19 countries, including all of those with autochthonous infections except Serbia (Table 1).

Antibody tests, including the immunofluorescence antibody test, ELISA, and the rapid immunochromatography test, are the main surveillance diagnostic methods used, followed by PCR. Antibody tests play a fundamental role in disease surveillance because they are relatively cheap and easy to use (10). However, their sensitivity to detect subclinical infections is lower than that of PCR tests (10), and they do not discriminate naturally infected from vaccinated dogs (11). PCR tests are ideal for epidemiologic studies to estimate Leishmania spp. infection prevalence in healthy hosts, but their diagnostic validity depends on the sample used, the DNA sequence target, and the PCR protocol. Standardization of PCR tests in leishmaniasis diagnosis is needed (12).

Of the 7 countries that have ongoing AniL prevention and control programs (Table 1), 5 use topical insecticides for dogs, 5 are diagnosing and treating leishmaniases in dogs, and 2 use canine leishmaniosis vaccines. In all countries, infected dogs may be euthanized on welfare grounds. Lack of funds and treatment costs were considered the most important AniL control challenges. Human leishmaniasis prevention and control activities are implemented in 12 countries (Table 1); for L. infantum, actions focused on the use of insecticides on dogs, and for L. major, L. tropica, and L. donovani, the common activity was the use of peridomiciliary and intradomiciliary insecticides. Lack of funds and capacity constraints are considered the main challenges for HumL.

Although zoonotic L. infantum strategies are centered on preventing and eliminating infections in dogs, the main parasite reservoir host, we found that insecticides and treatments are not fully effective and are expensive, and so provided to a relatively small proportion of dogs. Leishmaniasis control needs the One Health approach to account for the complexity of its transmission cycle involving humans, domestic animals, wildlife, and sand fly vectors (13).

Animal leishmaniases are considered emergent diseases in Cyprus and Jordan and in parts of Algeria, 
Table 1. Declared country status of leishmaniases surveillance and control, 2010-2020*

\begin{tabular}{|c|c|c|c|c|c|c|c|c|}
\hline \multirow[b]{2}{*}{ Country } & \multicolumn{2}{|c|}{ Autochthonous } & \multicolumn{2}{|c|}{ Notifiable } & \multicolumn{2}{|c|}{ Surveillance } & \multicolumn{2}{|c|}{ Control } \\
\hline & Animal & Human & Animal & Human & Animal & Human & Animal & Human \\
\hline Albania & NR & $\mathrm{VL}, \mathrm{CL}$ & NR & Yes & NR & Yes & NR & No \\
\hline Algeria & Yes & NR & Yes & NR & Yes & NR & Yes & NR \\
\hline Armenia & Yes & VL & Yes & Yes & Yes & Yes & Yes & Yes \\
\hline Austria & Not known & No & No & No & No & No & No & No \\
\hline Azerbaijan & NR & VL, CL & NR & Yes & NR & Yes & NR & Yes \\
\hline Belgium & No & No & No & No & No & Yes & No & No \\
\hline $\begin{array}{l}\text { Bosnia and } \\
\text { Herzegovina }\end{array}$ & No & NR & Yes & NR & No & NR & No & NR \\
\hline Bulgaria & NR & VL & NR & Yes & NR & Yes & NR & Yes \\
\hline Croatia & Yes & VL, CL & Yes & Yes & No & Yes & No & Not known \\
\hline Cyprus & Yes & VL, CL & Yes & Yes & Yes & Yes & No & No \\
\hline Czechia & Not known & No & Yes & Yes & No & No & No & No \\
\hline France & Yes & VL, CL & No & No & No & Yes & No & No \\
\hline Georgia & Yes & VL & Yes & Yes & No & Yes & No & Yes \\
\hline Germany & Not known & No & No & No & No & No & No & No \\
\hline Greece & Yes & VL, CL & Yes & Yes & No & Yes & Yes & Yes \\
\hline Hungary & Not known & NR & No & NR & No & NR & No & NR \\
\hline Israel & Yes & VL, CL & Yes & Yes & No & Yes & No & Yes \\
\hline Italy & Yes & VL, CL & Yes & Yes & Yes & Yes & Yes & Yes \\
\hline Jordan & Yes & NR & Yes & NR & No & NR & No & NR \\
\hline Libya & NR & VL, CL & NR & Yes & NR & Yes & NR & Yes \\
\hline Luxemburg & Not known & NR & No & NR & No & NR & No & NR \\
\hline Malta & NR & VL, CL & NR & Yes & NR & Yes & NR & Yes \\
\hline Moldova & No & NR & Yes & NR & No & NR & No & NR \\
\hline Montenegro & No & VL & Yes & Yes & No & Yes & Yes & No \\
\hline North Macedonia & Yes & NR & Yes & NR & Yes & NR & Yes & NR \\
\hline Palestine & Yes & NR & No & NR & No & NR & Yes & NR \\
\hline Romania & Yes & No & No & Yes & No & No & No & Not known \\
\hline Serbia & Yes & $\mathrm{VL}, \mathrm{CL}$ & No & No & No & No & No & Yes \\
\hline Slovenia & Yes & No & Yes & Yes & No & Yes & No & No \\
\hline Spain & Yes & VL, CL & Regionally & Yes & Yes & Yes & No & Yes \\
\hline Turkey & Yes & $\mathrm{VL}, \mathrm{CL}$ & No & Yes & No & Yes & No & Yes \\
\hline Ukraine & Yes & $\mathrm{VL}$ & Regionally & Yes & Yes & Yes & No & No \\
\hline
\end{tabular}

Armenia, France, Georgia, Jordan, Montenegro, North Macedonia, Romania, Slovenia, Turkey, and Ukraine. The most important AniL emergence risk factor is the lack of control. Human leishmaniases are considered emerging diseases in Cyprus, Libya and Malta and in parts of Albania, Austria, Armenia, Azerbaijan, Georgia, Israel, Italy, Montenegro, and Spain. The main risk factors for HumL emergence are vector expansion for L. infantum, and movement of infected persons between countries for L. major, L. tropica, and L. donovani.

In general, the perceived increasing risk for AniL and HumL was in line with the literature. In the EU and its neighborhood, the risks include movement of humans and dogs, increased number of immunosuppressed patients, climate warming, and other environmental changes affecting vector and reservoir host distribution $(1,14)$. Limitations associated with existing surveillance and control programs, along with the fact that leishmaniases are often regarded as a local problem rather than a transnational problem, are deemed major obstacles to overcome to prevent leishmaniases emergence in the EU and its neighborhood.

\section{Conclusions}

Leishmaniases are considered widespread, endemic, or emerging infections in the EU and its neighborhood, yet are neglected and underreported because they are low priority at the country and EU level. Our study revealed a clear need to strengthen leishmaniasis prevention and control programs in the EU and its neighborhood. We recommend analysis of leishmaniasis incidence in the region for an objective assessment of disease emergence, and also improvement of prevention and control programs based on a robust surveillance and following a One Health approach.

\section{Acknowledgments}

We thank experts from the public health institutes, animal health institutes, ministries of health, and ministries of agriculture from Albania, Algeria, Armenia, Austria, Azerbaijan, Belgium, Bosnia and Herzegovina, Bulgaria, Croatia, Cyprus, Czechia, France, Georgia, Germany, Greece, Hungary, Israel, Italy, Jordan, Libya, Luxembourg, Malta, Moldova, Montenegro, North Macedonia, Palestine, Romania, Serbia, Slovenia, Spain, Turkey, and Ukraine for 
taking the time to answer the questionnaires and providing us the information used to prepare this review on leishmaniasis. In particular, we thank: Silva Bino, Adela Vasili and Teita Myrseli (Albania), Ahmed Chawki El Karim Boughalem (Algeria), Arman Gevoryan, Lusine Paronyan and Narek Hayrapetyan (Armenia), Irene Kászoni-Rückerl and Julia Walochnik (Austria), Yagut
Garayeva (Azerbaijan), Javiera Rebolledo (Belgium), Aleksandar Nemet (Bosnia and Herzegovina), Rumen Harizanov (Bulgaria), Tihana Miškić, Ivana Lohman Janković and Eddy Listeš (Croatia), Maria G. Koliou and Vasiliki Christodoulou (Cyprus), Jerome Depaquit, Laurence Lachaud, Christophe Ravel and Patrick Bastien (France), Merab Iosava and Tegniz Chaligava (Georgia),

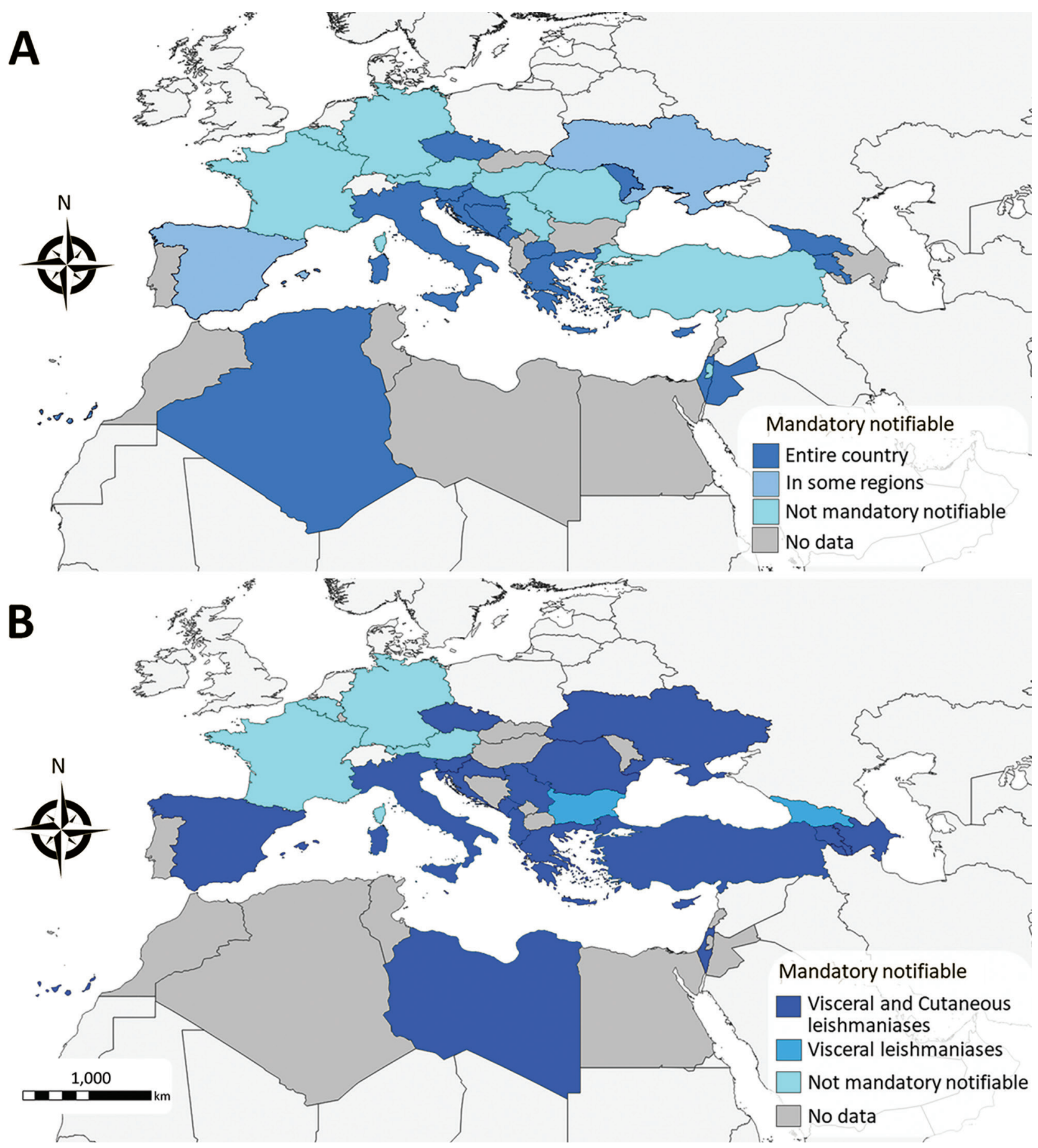

Figure 2. Geographic distribution of mandatory notification status for animal (A) and human (B) leishmaniases, 2020. 
Table 2. Declared status of endemicity of Leishmania spp. affecting humans, by country

\begin{tabular}{|c|c|c|c|c|}
\hline \multirow[b]{2}{*}{ Country } & \multicolumn{4}{|c|}{ Leishmania species } \\
\hline & L. infantum & L. major & L. tropica & L. donovan \\
\hline Albania & Yes & No & No & No \\
\hline Armenia & Yes & No & No & No \\
\hline Austria & No & No & No & No \\
\hline Azerbaijan & Yes & Yes & Yes & No \\
\hline Belgium & No & No & No & No \\
\hline Bulgaria & Yes & No & No & No \\
\hline Croatia & Not known & Not known & Not known & Not known \\
\hline Cyprus & No & No & Yes & Yes \\
\hline Czechia & No & No & No & No \\
\hline France & Yes & No & No & No \\
\hline Georgia & Yes & Yes & No & No \\
\hline Germany & No & No & No & No \\
\hline Greece & Yes & No & No & No \\
\hline Israel & Yes & Yes & Yes & No \\
\hline Italy & Yes & No & No & No \\
\hline Libya & Yes & Yes & Yes & Not known \\
\hline Malta & Yes & No & No & No \\
\hline Montenegro & Yes & Not known & Not known & No \\
\hline Romania & No & No & No & No \\
\hline Serbia & Yes & Not known & Yes & No \\
\hline Slovenia & No & No & No & No \\
\hline Spain & Yes & No & No & No \\
\hline Turkey & Not known & Not known & Yes & Not known \\
\hline Ukraine & No & No & No & No \\
\hline
\end{tabular}

Franz J. Conraths (Germany), Danai Pervanidou and Michail Floros (Greece), Tamás Sréter (Hungary), Emilia Anis, Roee Singer, Yael Glazer and Michel Bellaiche (Israel), Alda Natale, Gianluca Rugna, Mose' Alise, Patrizia Parodi, Luigi Gradoni and Pellegrino Daniele (Italy), Mahmoud Alhanatleh (Jordan), Badereddin Annajar (Libya), Maxim Sirbu (Moldova), Mevlida Hrapovic and Nebojša Sekulić (Montenegro), Iyad Adra (Palestine), Alexandru Supeanu and Cristina Daniela Pop (Romania), Mitra Drakulovic and Sasa Ostojic (Serbia), Maja Sočan (Slovenia), Beatriz Fernández Martínez, Francisco Javier Moreno Nuncio, Francisco Javier Nieto Martínez, Soledad Collado Cortés, Jose Luis Sáez Llorente and Alejandro Pérez Riquelme (Spain), Anil Demeli, Ahmet Deniz and Seher Topluoglu (Turkey) and Ihor Kuzin (Ukraine). In addition, we thank Tamás Bakonyi for reviewing and testing the questionnaire.

Y.V.d.S. is employed with the European Food Safety Authority (EFSA) in the ALPHA Unit that provides scientific and administrative support to EFSA's scientific activities in the area of Animal Health and Welfare. The positions and opinions presented in this article are those of the authors alone and are not intended to represent the views or scientific work of EFSA.

\section{About the Author}

Dr. Berriatua is a professor of animal health at the University of Murcia in Spain, where he teaches and conducts research on the epidemiology and ecology of parasitic diseases and specifically leishmaniases.

\section{References}

1. Gradoni 1. the leishmaniases of the Mediterranean region. Curr Trop Med Rep. 2017;4:21-6. https:/ / doi.org/ 10.1007/s40475-017-0099-1

2. European Centre for Disease Prevention and Control. VectorNet, Phlebotomine sandflies maps. 2021 [cited 2021 Mar 19]. https:/ / www.ecdc.europa.eu/en/disease-vectors/ surveillance-and-disease-data/phlebotomine-maps

3. European Commission. EUSurvey, version 1.5. 2021 [cited 2021 Mar 15]. https://ec.europa.eu/eusurvey/home/ welcome

4. Colella V, Hodžić A, Iatta R, Baneth G, Alić A, Otranto D. Zoonotic leishmaniasis, Bosnia and Herzegovina. Emerg Infect Dis. 2019;25:385-6. https://doi.org/10.3201/ eid2502.181481

5. Tánczos B, Balogh N, Király L, Biksi I, Szeredi L, Gyurkovsky M, et al. First record of autochthonous canine leishmaniasis in Hungary. Vector Borne Zoonotic Dis. 2012;12:588-94. https://doi.org/10.1089/ vbz.2011.0906

6. Hamarsheh O, Nasereddin A, Damaj S, Sawalha S, Al-Jawabreh H, Azmi K, et al. Serological and molecular survey of Leishmania parasites in apparently healthy dogs in the West Bank, Palestine. Parasit Vectors. 2012;5:183. https://doi.org/10.1186/1756-3305-5-183

7. Ozbel Y, Oskam L, Ozensoy S, Turgay N, Alkan MZ, Jaffe CL, et al. A survey on canine leishmaniasis in western Turkey by parasite, DNA, and antibody detection assays. Acta Trop. 2000;74:1-6. https:/ / doi.org/10.1016/ S0001-706X(99)00047-9

8. Lachaud L, Dedet JP, Marty P, Faraut F, Buffet P, Gangneux JP, et al.; Working Group for the Notification of Human Leishmanioses in France. Surveillance of leishmaniases in France, 1999 to 2012. Euro Surveill. 2013;18:20534. https://doi.org/10.2807/1560-7917. ES2013.18.29.20534

9. Carrillo E, Moreno J, Cruz I. What is responsible for a large and unusual outbreak of leishmaniasis in Madrid? Trends Parasitol. 2013;29:579-80. https://doi.org/10.1016/ j.pt.2013.10.007

10. Thakur S, Joshi J, Kaur S. Leishmaniasis diagnosis: an update on the use of parasitological, immunological, and molecular methods. J Parasit Dis. 2020 Mar 16; 44:1-20.

11. Solano-Gallego L, Cardoso L, Pennisi MG, Petersen C, Bourdeau P, Oliva G, et al. Diagnostic challenges in the era of canine Leishmania infantum vaccines. Trends Parasitol. 2017;33:706-17. https:/ / doi.org/10.1016/j.pt.2017.06.004

12. Varani S, Ortalli M, Attard L, Vanino E, Gaibani P, Vocale C, et al. Serological and molecular tools to diagnose visceral leishmaniasis: 2-years' experience of a single center in Northern Italy. PLoS One. 2017;12:e0183699. https://doi.org/10.1371/journal.pone.0183699

13. Palatnik-de-Sousa CB, Day MJ. One Health: the global challenge of epidemic and endemic leishmaniasis. Parasit Vectors. 2011;4:197. https:/ / doi.org/10.1186/ 1756-3305-4-197

14. Wright I, Jongejan F, Marcondes M, Peregrine A, Baneth G, Bourdeau P, et al. Parasites and vector-borne diseases disseminated by rehomed dogs. Parasit Vectors. 2020;13:546. https:// doi.org/10.1186/s13071-020-04407-5

Address for correspondence: Céline M. Gossner, European Centre for Disease Prevention and Control, Gustav III:s Boulevard 40, 168 73 Solna, Sweden; email: celine.gossner@ecdc.europa.eu 